\title{
Der literarische Pakt. Lesen in Alexander Kluges Die
} Lücke, die der Teufel läßt

Le pacte littéraire. Lire dans Die Lücke, die der Teufel läßt d'Alexander Kluge The literary contract. Reading in Alexander Kluge's Die Lücke, die der Teufel läßt (The Gap left by the Devil)

\section{Susanne Marten}

\section{CpenEdition}

\section{Journals}

\section{Édition électronique}

URL : http://journals.openedition.org/ceg/1158

DOI : $10.4000 /$ ceg. 1158

ISSN : 2605-8359

\section{Éditeur}

Presses Universitaires de Provence

\section{Édition imprimée}

Date de publication : 18 décembre 2015

Pagination : 117-126

ISBN : 979-1-03200-020-5

ISSN : 0751-4239

\section{Référence électronique}

Susanne Marten, "Der literarische Pakt. Lesen in Alexander Kluges Die Lücke, die der Teufel läßt», Cahiers d'Études Germaniques [Online], 69 | 2015, Online erschienen am: 17 Dezember 2017, abgerufen am 27 November 2020. URL : http://journals.openedition.org/ceg/1158 ; DOI : https://doi.org/ $10.4000 /$ ceg. 1158 


\section{Der literarische Pakt. Lesen in Alexander Kluges Die Lücke, die der Teufel läßt}

Susanne MARTEN

Université de Strasbourg

\section{Die Teile und das Ganze}

Um die Schwelle zu senken, sich einen Weg durch Die Lücke, die der Teufel läßt zu bahnen, kündigt sein Autor bzw. der Erzähler gleich im Vorwort an: „Jedem Kapitel dieses Buches gehen Zeilen voran, aus denen der Leser sich orientieren kann, welche Kapitel sein Interesse wecken. " ${ }^{1}$ Es wird also niemandem zugemutet, eine kontinuierliche, lineare Lektüre zu bewältigen. In der Tat sind dafür nicht nur die Geschichten zu zahlreich, etwa fünfhundert sind es auf fast tausend Seiten, auch die Themen scheinen, zumindest auf den ersten und zweiten Blick, zu disparat für eine zusammenhängende Lektüre. Berechtigt mag allerdings die Frage sein, warum dann die Geschichten in diesem dicken, widerständigen Buch quasi vergraben wurden, und ob es nicht wünschenswert wäre, sie an unscheinbaren, aber mühelos zugänglichen Orten, z.B. in der Tagespresse, finden zu können. ${ }^{2}$ Wäre dem so, könnte man hinter dem Buch Die Lücke, die der Teufel läßt eine reine Sammlung von Geschichten vermuten, nach Art des ,Schatzkästleins“, oder ein Brevier, in dem - zur Erbauung, und je nach Geschmack, Bedürfnis und Tagesform - jeder etwas zu finden vermöchte. In diesem Sinne richtete Alexander Kluge schon in seiner drei Jahre zuvor erschienenen Chronik der Gefühle eine nachdrückliche Einladung an die Leserschaft „wie bei einem Kalender oder eben einer Chronik“ nachzuprüfen, was sie betreffe, denn „niemand wird so viele Seiten auf einen Schlag lesen“" Zugleich versprechen die ermunternden Worte aus der Einleitung von Die Lücke, die der Teufel läßt gerade keine lehrreiche Lektüre, die der Introspektion oder der Prüfung des eigenen Gewissens dienen könnte, sondern betonen, das Buch lenke den Blick auf Orientierung in der faktischen Realität. Auch auf stilistischer Ebene findet eine Abkehr von Chronik und Kalender statt. Erläuternde Zeilen zu Beginn eines

Alexander Kluge, Die Lücke, die der Teufel läßt, Frankfurt a. M., Suhrkamp, 2003, S. 8.

2 Dazu fand auch eine Diskussion während der Wiener Tagung Die Frage des Zusammenhangs im Oktober 2010 statt, die Rainer Stollmann mit dieser zugespitzten Formulierung anregte.

3 Alexander Kluge, Chronik der Gefühle. Basisgeschichten, Bd. I, Frankfurt a. M., Suhrkamp, 2000, S. 7. 
jeden Kapitels evozieren in der Tat eher das Volksbuch bzw. den Prosaroman ${ }^{4}$ der frühen Neuzeit, wie beispielsweise den Fortunatus oder die Historia von Dr. Johann Fausten, aber auch den Schwankroman Eulenspiegel, oder das Lalebuch, die späteren Schildbürger-Streiche. Solch einer Historiensammlung ohne Held gleicht Die Lücke auch durch ihre offene Form und die scheinbar lose Reihung von Begebenheiten.

Die Einladung, einzelne Kapitel in der Lücke zu entdecken, scheint mir deshalb vor allem ein Angebot, es mit dem Buch doch erst einmal zu versuchen, verhüllt in eine rhetorische Bescheidenheitsformel, die wiederum die Literarizität des Werkes unterstreicht. Dass damit zugleich eine Einladung zum Wildern verbunden ist, also an den Leser die Aufforderung ergeht, die interessantesten oder spannendsten Kapitel aus dem Buch selbst herauszugreifen, bestätigt Kluges Kommentar zu seinem Nachweise und Hinweise ${ }^{5}$ überschriebenen Handapparat: „Man wühlt gewissermaßen einmal quer." 6

Im Gegensatz zu Alexander Kluge, dem leserfreundlichen Vorwortschreiber der Lücke, geht der Interpret Kluge jedoch noch einen Schritt weiter, wenn er darüber hinaus anregt, sein Buch motivisch zu lesen und zu ordnen: „Wenn Sie nichts weiter täten, als in dem Buch alle Flüsse, alle Behauptungen und Erzählungen übers Wasser zusammen zu greifen, hätten Sie eine eigene Geschichte. Dasselbe könnten Sie mit der Wilden Jagd der Geister machen." Kontext des gesamten Buches bliebe jede einzelne Erzählung, jedes Kapitel, für sich genommen, unvollständig. Doch auch der Vorschlag, Motivketten durch solches Zusammengreifen zu bilden, erweist sich als spannungsvoller angelegt denn zunächst vermutet, weil Kluge sein literarisches Werk zugleich zwischen den Polen Gegensatz und Ganzheit verortet: „Die Geschichten bilden untereinander Gegensätze oder Konkurrenzen, manchmal ergeben sie zusammen ein Full House. Nicht mit einem Handlungsraster, einem Handlungszwang." 8 An der kritischen Theorie und Theodor W. Adorno, und damit indirekt zugleich an Nietzsche geschult, unterstreicht Kluge die Zusammengehörigkeit von Gegensätzen ${ }^{9}$ und deutet an, dass erst durch ein konstellatives Zusammenstehen ${ }^{10}$ die Geschichten, und damit die Problematik, die sie bergen, sichtbar würden. Fast im gleichen Atemzug nennt Kluge als weitere Möglichkeit nämlich die Abkehr von der sprachlichen Darstellung selber, und fasst einen Medienwechsel ins Auge: „Darüber [nämlich

4 Zur Begrifflichkeit siehe Jan-Dirk Müller, Volksbuch/Prosaroman. Perspektiven der Forschung, in Internationales Archiv für Sozialgeschichte der deutschen Literatur (IASL) 1, Sonderheft 1985, S. 1-128.

5 Kluge, Die Lücke, S. 909-929.

6 Kluge im Interview mit Claus Philipp, „Erzählungen haben den Vorteil, dass sie blind sein dürfen“, in Volltext, 15.10.2003, URL: http://volltext.net/magazin/magazindetail/article/149 [Stand: 15.5.2015].

7 Ibid.

8 Ibid.

9 Als Frage formuliert: „Was zwingt uns überhaupt zur Annahme, dass es einen wesenhaften Gegensatz von ,wahr' und ,falsch“ giebt?“, Friedrich Nietzsche, Jenseits von Gut und Böse, Kritische Studienausgabe Bd. 5, hrsg. von Giorgio Colli und Mazzino Montinari, München/Berlin, dtv/de Gruyter, 1988, S. 53, s. auch S. 16.

10 „Der Konstellation gewahr werden, in der die Sache steht, heißt soviel wie diejenige entziffern, die es als Gewordenes in sich trägt", Theodor W. Adorno, Negative Dialektik, Gesammelte Schriften 6, hrsg. von Rolf Tiedemann, Frankfurt a. M., Suhrkamp, 1998 [1970], S. 165. 
über Motive, Motivketten, Zusammenhänge, Anm. d. Verf.] Karten zu haben, Informationen, Navigationsmöglichkeiten, das ist ein Gebrauchswert eines solchen Buches in meinen Augen." ${ }^{11}$ Nicht nur wird also zu einer Lesart angeregt, die vom close-reading bis zum Überblick reichen kann. Auch unter allen drei ästhetischen Gesichtspunkten, dem Medienwechsel, der schriftstellerischen Produktion sowie der Rezeption, so betont es Kluge, hängen die Geschichten zusammen und der Weg kann jeweils vom Text zur Karte bzw. zum Netz, von divergenten Motiven zur strukturierten Information, und schließlich vom „wühlenden“ Lesen hin zur zielgerichteten Navigation verlaufen.

Man könnte also versuchen, über die Erzählungen und Kapitel hinaus, aber auch jenseits von vereinzelten Motivreihen, Die Lücke, die der Teufel läßt als Ganzes in den Blick zu bekommen. Dabei könnten sich nicht nur neue Horizonte, oder neue Untiefen auftun, die den besonderen Stellenwert des Buches im Gesamtzusammenhang des Werkes begründen, oder Elemente zu einem neuen Verständnis von Alexander Kluges Werk liefern. Karten über noch unbekanntes doch navigierbares Gebiet, um in Kluges Metapher zu bleiben, sind eine Aufforderung, neue Routen zu erkunden, wo nicht ein ausdrückliches Versprechen auf philosophische Erkenntnis. Der Frage wäre nachzugehen, ob sich ein klugegerechtes distant-reading ${ }^{12}$ erfinden lässt. Es bietet sich auf jeden Fall an, Kluges Geschichten mit Kluge auch gegen Kluge zu lesen und den Gedanken eines möglichen Zusammenhangs der Geschichten nicht gleich von der Hand zu weisen. Ob dieser Weg dann notwendigerweise über einen Medienwechsel führen muss ${ }^{13}$, sei erst einmal dahingestellt.

\section{Geschichte und Geschichten}

Obwohl Die Lücke, die der Teufel läßt, im Herbst 2003 erschienen, geschichtlich weit ausgreift, ist das Buch dennoch stark in der Gegenwart verortet. Im engeren Sinne heißt das zunächst im Jahr 2003, oder genauer, in den Monaten nach dem 11. September 2001. ${ }^{14}$ Die eingestürzten Türme des New Yorker World Trade Center finden sich denn nicht nur in Form von scheinbar Nebensachen (beispielsweise Versicherungsfragen) behandelnden Geschichten in der Lücke, sondern auch als

11 Kluge, Interview mit Claus Philipp.

12 Einen quantitativen Ansatz verfolgt Gunther Martens: s. z.B. „Distantly reading Alexander Kluge's Distant Writing“, in Vermischte Nachrichten. Alexander Kluge Jahrbuch 1, Göttingen, Vandenhoeck \& Ruprecht, 2014, S. 29-41. Zum distant reading, s. Franco Moretti, Graphs, Maps, Trees. Abstract Models for a Literary History, London, Verso, 2005 und ders., Distant Reading, London,Verso, 2013, sowie Matt Erlin/Lynne Tatlock (Hrsg.), Distant Readings - Descriptive Turns. Topologies of German Culture in the Long Nineteenth Century, Rochester, Camden House, 2014.

${ }^{13}$ In seiner Rezension von Kluges Das fünfte Buch vermerkt Florian Felix Weyh, das Buchformat entspreche nicht länger dessen von Aktualität geprägten Kurztexten. Die Parallele, die er zur „Patchworkinformation in sozialen Netzwerken“ zieht, dient ihm jedoch eher der ästhetischen Kritik als einer fundierten Mediendiskussion: „Für Kluge Leser“, Deutschlandfunk, 19.02.2012. URL: http://www. deutschlandfunk.de/fuer-Kluge-leser.700.de/html?dram:article_id=85415 [Stand: 15.5.2015].

${ }^{14}$ Siehe auch Rainer Stollmann, Die Entstehung des Schönheitssinns aus dem Eis. Gespräche mit Alexander Kluge, Berlin, Kadmos, 2006, S. 115. 
Cover-Illustration ${ }^{15}$ auf dem kartonierten Schuber. Gegenwart heißt aber auch: nach dem Zusammenbruch des sowjetischen Imperiums. Der Fall der Berliner Mauer und die Vereinigung der beiden Deutschlands im Jahr 1990 stellen zwar einen wichtigen Aspekt davon dar, weitaus prägender wirkten sich aber das Ende des Kalten Krieges und die neuen Funktionalitäten und dysfunktionalen Strukturen aus. Von dieser (vergangenen) Gegenwart her richtet sich der Blick auf das Jahrzehnt zwischen 1991 und 2001, in dem der Beginn unserer heutigen Globalisierung liegt, und in dem unbemerkte Veränderungen stattgefunden haben, die den elften September noch heute als Wendepunkt erscheinen lassen. Vor den genannten Daten, also vor dem Ende des Kalten Krieges, vor 1991, liegt die Geschichte des zwanzigsten Jahrhunderts, markiert durch Studentenproteste, Kriegsende, Nationalsozialismus, Weimar, Ersten Weltkrieg. Dennoch ist Die Lücke, die der Teufel läßt kein Geschichtsbuch, das sich allein an historisch bedeutsamen Jahreszahlen orientieren würde. Kluges Erzählungen berichten auch von sehr alltäglichen Situationen in außergewöhnlichen Zeiten, ebenso wie von „unerhörten Begebenheiten“ ${ }^{16}$ in scheinbar bewegungslosem Umfeld. Das bedeutet beispielsweise, dass „Zwischenzeiten“ ${ }^{17}$ mindestens ebenso bedeutungsvoll, wenn nicht wichtiger sind. Das dritte Kapitel, in dem die Stadt Paris im Juni 1940 zum Protagonisten und Anti-Helden wird, bezieht sich auf die geschichtliche Konstellation einer Zeit, die zumindest im kollektiven Gedächtnis der Deutschen keine Rolle spielt. Kluge bezeichnet diese Zeiten unter Verwendung des physikalischen Begriffs als „ABARISCHE PUNKTE, also Punkte außerhalb der wahren Schwereverhältnisse der Welt" ${ }^{\text {"18 }}$, die innerhalb und zugleich außerhalb des geschichtlichen Zusammenhangs existieren. Seine Bücher erzählen aber nicht vom Vergehenden, vielmehr versuchen sie das „Ahnungsvermögen“ inmitten des Vergangenen und Gegenwärtigen zu schärfen. Ohne dass Voraussagenüber die Zukunft getroffen würden, wird die erzählte Gegenwart so abgeklopft, dass auch Bereiche davon erklingen, die bisher noch nicht als geschichtlich gewordene wahrgenommen wurden. Kluge selbst beschreibt sein Erzählen im Bild der Fledermaus, die Signale aussendet, und anhand des Echos den sie umgebenden Raum ausmisst ${ }^{19}$, doch auch Nietzsches Philosophie mit dem Hammer, mit dem der Philosoph perkutierend die Gegenwart auf klingende Stellen abklopft, passt auf sein Verfahren: „Hier einmal mit dem Hammer Fragen stellen [...] wie mit einer Stimmgabel“"20. Auf diese Weise wäre Gegenwart nicht mehr allein eine Ansammlung von Geschichte, sondern die Membran, an der Zukunft sich ankündigt, die vom „Ahnungsvermögen“

${ }^{15}$ Das berühmte Foto von Steve McCurry (Magnum) wurde am Morgen des 12.09.2001 aufgenommen.

${ }^{16}$ Johann Wolfgang von Goethe, Sämtliche Werke. Briefe, Tagebücher und Gespräche, Bd. 12 (39): Johann Peter Eckermann, Gespräche mit Goethe in den letzten Jahren seines Lebens, hrsg. von Christoph Michel, Frankfurt a. M., Dt. Klassiker Verlag, 1999, S. 221. Gespräch vom 29. Januar 1827 über die Novelle.

${ }^{17}$ Kluge, Die Lücke, S. 222-234, s. auch Stollmann, Die Entstehung des Schönheitssinns, S. 25.

18 Stollmann, Die Entstehung des Schönheitssinns, S. 22, auch Die Lücke, S. 240.

19 Kluge im Interview mit Rainer Stollmann, „Intelligenz zwischen Teufelsforschung und Wertgesetz“, in Glossen 18/2003. URL: http://www2.dickinson.edu/glossen/heft18/alexander.Kluge. interview.html, 2003 [Stand: 15.5.2015].

${ }^{20}$ Friedrich Nietzsche, Götzendämmerung, Kritische Studienausgabe Bd. 6, S. $57 \mathrm{f}$. 
erhört werden kann. ${ }^{21}$ Für die Lücke, die der Teufel läßt gilt dies in besonderem Maße. Seit ihrer Veröffentlichung sind die Themen, die sich im Jahr 2003 erst andeuteten, vielfach Gegenstand genauer Untersuchungen geworden. Genannt seien beispielsweise die neue Rolle Russlands in der Weltpolitik, die „Festung Europa“ als Antwort auf Asylsuchende aber auch Enzensbergers Hammerstein oder Der Eigensinn $^{22}$, das im Jahr 2008 erschien und in dem Enzensberger nicht nur auf Kluge/Negts Werk Geschichte und Eigensinn ${ }^{23}$ Bezug nimmt, sondern auch explizit dessen Geschichte „Lebendigkeit von 1931“24 als eine seiner Inspirationsquellen nennt. Kluge gehe darin ,souverän, um nicht zu sagen skrupellos mit den Tatsachen um“, schreibt Enzensberger, und seine Zeilen enthalten eine Hommage an Kluges „phantastische Rekonstruktion historischer Augenblicke“ ${ }^{25}$.

So ist Die Lücke, die der Teufel läßt nicht nur phantastisches Geschichts- sondern auch ein ungehemmt erzählendes Geschichtenbuch, das noch eine besondere Herausforderung birgt, und zwar zunächst einmal die schiere Fülle und der Disparatheit der Geschichten. Wenn es im Gespräch um die tausend Seiten und die fünfhundertzweiundsiebzig Geschichten der Lücke geht, provoziert dies unfehlbar die Nachfrage, ob es da eigentlich irgendeinen Zusammenhang gebe. Der Versuch, sich erläuternd anhand der Kapitel und ihrer Überschriften voranzutasten, scheitert dann in der Regel schon im Ansatz. Wie soll man auch einen Zusammenhang erkennen bei Themen wie „Zwischen lebendig und tot/Was heißt lebendig?“", „Tschernobyl“, „Paris, Juni 1940“, „Die Mondkräfte und der Endsieg“, „U-Boot-Geschichten“, „Mit Haut und Haaren: Basisgeschichten“, „Wach sind nur die Geister“, „Die Schatzsucher“, „Festung Europa“, um nur einige zu zitieren? Zwar klingt das Thema des Todes in vielen Titeln an, und damit eng verknüpft, die Frage nach dem Überleben in einer unsicheren Welt, aber Kluge, gefragt, ob er mit seinen Geschichten keine Richtung verbinde, behauptet: „Mindestens die Hälfte der Geschichten hat einen glücklichen Ausgang. Gegen alle Wahrscheinlichkeit." ${ }^{26}$ Um einen Abgesang auf die westliche Zivilisation kann es sich also auch nicht handeln. Kluges eigene Aussage, er habe auf den „Rohstoff“ 27 der Literatur zurückgehen wollen, ist im Zusammenhang mit seinem Erzähltempo zu sehen, schnell und vieles aus der Jetztzeit erzählen zu wollen, bedeutet aber nicht, dass er statt zu dichten eine Sammlung von Dokumenten anlegen würde. Die Lücke, die der Teufel läßt, wie Kluges andere Bücher, lässt sich auch nicht auf ihren Inhalt, ihren plot, reduzieren. Mag die Themenvielfalt unübersichtlich und von immenser Fülle sein, stets herrscht der gleiche lakonische

${ }^{21}$ Dass Kluge keinem Vorhersagespiritismus das Wort redet, dafür bürgt folgender Dialog aus „Hitler als ,Mondgänger““: „Ahnungsvermögen oder Instinkt. [...] Beispiele? [...] 7140 [...] Irrtümer? [...] Die Wiederholung des gleichen Irrtums habe ich abgezogen, sonst wären es 3192.“, in Kluge, Chronik, S. 40f.

${ }^{22}$ Hans Magnus Enzensberger, Hammerstein oder Der Eigensinn, Frankfurt a. M., Suhrkamp, 2008, s. auch Die Lücke, S. 25f.

23 Alexander Kluge/Oskar Negt, Geschichte und Eigensinn, Frankfurt a. M., Zweitausendeins, 2001 [Suhrkamp, 1993].

${ }^{24}$ Kluge, „Lebendigkeit von 1931“, in Die Lücke, S. 25-30.

25 Enzensberger, Hammerstein, S. 354.

${ }^{26}$ Alexander Kluge im Interview mit Ulrich Greiner und Iris Radisch, „Der Friedensstifter“, in Die Zeit, Nr. 44, 23.10.2003. URL: http://www.zeit.de/2003/44/L-Kluge [Stand 15.5.2015].

27 Ibid. 
Ton, der die literarische Sprache der Darstellung eines juristischen Sachverhalts, einem Protokoll oder auch dem lapidaren Gestus einer Märchenerzählung annähert. Statt dass Spannung entstünde, werden Spannungsmomente durch Dehnung oder präzise Beschreibungen abgebaut oder umgangen. Das erzeugt schon bei kurzer Lektüre ein eigenartiges Befremden und wirft eine Fülle an Fragen auf. Handelt es sich um wahre Begebenheiten, oder sind die Erzählungen Fiktion? Kann man die teils skurrilen Figuren und Ereignisse ernst nehmen, oder ist die Geschichte ironisch gemeint? Sind die stilistischen Anklänge, an Kleist zum Beispiel, Parodie, oder doch etwas anderes?

Die Bedeutung, die er der literarischen Sammlung beimisst, unterstreicht Alexander Kluge in seinem Buch selber, indem er betont, dass sie „eine neue Literaturgattung darstellt und für Texte des 21. Jahrhunderts eine Perspektive eröffnet" ${ }^{28}$, aber auch eine synthetische Arbeitsleistung, die noch vom Leser zu erbringen ist: „Mehr als die Chance, sich selbständig zu verhalten, gibt kein Buch. " ${ }^{29}$ Lässt man sich darauf ein, besteht viel Raum, in Die Lücke, die der Teufel läßt einzusteigen, ohne gleich darin zu verschwinden.

\section{Die Lücke, die der Teufel läßt: Buch, Kapitel, Erzählung - ein Zusammenhang?}

Unter dem Titel „Die Mondkräfte und der Endsieg/Die Lücke, die der Teufel läßt“", wird im vierten Kapitel die Neugier geweckt, nicht zuletzt durch die provokante Frage: „Befinden sich Menschen auf der Höhe ihrer Bösartigkeit?“30 Diese Frage wird zum Prüfstein in der Zeit nach den Anschlägen, in der sich allenthalben Spuren eines fundamentalistisch-religiösen Weltbildes zeigen, vor allem in G. W. Bushs Worten „die Welt vom Bösen befreien“, von der „Achse des Bösen“31 ebenso wie in seinem drohenden Sophismus, „wer nicht mit uns ist, ist gegen uns“ ${ }^{\text {“2 }}$. Während im neunten Kapitel unter dem Titel „Grand Guignol“"33 das scheinbar Böse als Lust, Unterhaltung und absichtsloses Glück dargestellt und entschärft ist, verfolgen die Geschichten im vierten Kapitel das objektive menschliche Interesse bis in die äußersten Verzweigungen der Subjektivität hinein: Kapitel 4 könnte man auch als das Gelehrten- und Forscherkapitel bezeichnen. Im Wesentlichen besteht es aus zwei Teilen, im ersten finden sich Geschichten, die von metaphysischer Spekulation, auszudeutenden Zeichen und historischen Umbrüchen erzählen. Deren

${ }^{28}$ Kluge, Die Lücke, (Anm. zu S. 199). Zum Thema des Erzählers als Sammler vgl. Wolfgang Reichmann, Der Chronist Alexander Kluge. Poetik und Erzählstrategien, Bielefeld, Aisthesis, 2009, S. 17-29.

${ }^{29}$ Oskar Negt/Alexander Kluge, Der unterschätzte Mensch, Bd. II: Geschichte und Eigensinn, Frankfurt a. M., Zweitausendeins, 2001, S. 5.

${ }^{30}$ Kluge, Die Lücke, S. 255.

${ }^{31}$ G.W. Bush, Rede in der National Cathedral, Washington, 14. September 2001, Pressemitteilung des Weißen Hauses, „Axis of Evil-Rede“, 29. Januar 2002. Siehe zu Kluges Horchen auf die Gegenwart auch Kluge/Stollmann, ,Intelligenz zwischen Teufelsforschung“.

32 Ibid., siehe Matthäus 12, 30: „Wer nicht mit mir ist, der ist wider mich“.

${ }^{33}$ Kluge, Die Lücke, „Grand Guignol“ (im Kapitel 9/2, „Mann ohne Kopf“), S. 693-706. 
Spannbreite reicht zum einen biographisch von der Thanatologie bis zur Frage der Abkunft, dann aber auch historisch von der Lutherzeit bis zum heutigen Stanford, und metaphysisch von christlichen Zeugnissen des zweiten Jahrhunderts über den klassischen Islam und die Kabbala bis zur Frage, welche Rolle der Religiosität im 21. Jahrhundert zukommen kann. ${ }^{34}$ Der gedankliche Hintergrund dieses ersten Teils wäre dann auch am ehesten der politischen Theologie zuzuordnen. Den gemeinsamen Nenner des zweiten Teils bildet Forschung im weitesten Sinne, aber auch Ernst Jüngersche Pseudo-Etymologie oder theoretische und praktische Forschung in biotechnologischen Laboren, vornehmlich solchen der NS-Zeit, doch auch in den aus ihnen hervorgegangenen Tochterinstituten. ${ }^{35}$

In der ersten Erzählung „Die Lücke, die der Teufel läßt“" ${ }^{\text {" }}$, titelgebend für Werk wie Kapitel, geht es um einen Inquisitionsprozess, in dem Ankläger und Verteidiger einer jungen als Halbhexe bezeichneten Frau, Annie Kerklaus, miteinander um Deutungshoheit über deren körperlichen Zusammenbruch ringen. Da dieser erst drei Stunden nach dem Ende der Folter erfolgt war, wird die Debatte grundsätzlich. Dem Ankläger Eckholt, der die Angeklagte aufgrund ihres Tränenausbruchs verurteilt wissen will, steht Dr. Ebner gegenüber, Arzt und Verteidiger, der sich weigert, die Tränen als Zeichen eines teuflischen Einflusses zu deuten. Die Natur, meint er, habe sich durchgesetzt und begibt sich mit dieser Aussage in Gefahr selbst verurteilt zu werden, denn diese ,gerade ist ja die Domäne des Teufels“, so sein Kontrahent Eckholt. Letzterer wird sich nach schließlich verlorenem Prozess unverzüglich rächen: „Dr. Ebner wurde auf Betreiben des sich verletzt fühlenden Dr. Eckholt am folgenden Morgen verhaftet, in Ingolstadt eingekerkert und angeklagt“‘.

\section{Ebner (Planities) stand...}

In seinem profunden Kommentar ${ }^{37} \mathrm{zu}$ dieser Geschichte arbeitet Joseph Vogl begrifflich heraus, wie die akademischen Disziplinen Theologie, Jurisprudenz und Medizin und deren divergierende Techniken und Deutungen miteinander verschränkt sind, und wie sie in komplexen „Wahrheitsspielen“ ihre Einsätze, und damit ihre Macht, gegeneinander verteidigen. Indem Gedanken ausgesprochen, also geordnet werden, schwächt sich dieser Anspruch im Verlauf des Prozesses zu Aussagen von lediglich höchstmöglicher Wahrscheinlichkeit ab. In den Mittelpunkt der Erzählung rückt dadurch das Unentscheidbare, das subjektiv (als „intentionslose Seinsweise“), historisch (als „Lücke im Drucksystem der Mächte“ ${ }^{38}$ ) und poetologisch (durch

\footnotetext{
${ }^{34}$ Kluge, Die Lücke, alle Themen in Erzählungen im ersten Teil des vierten Kapitels, S. 259-292.

35 „,Verlust der Jugend“, „,Eine Bemerkung von Ernst Jünger“, Die Lücke, S. 294; , ,Unbeherrschbarkeit des Abwehrreflexes von Muskeln im Augenblick der Tötung (1941)“, S. 300 ; „Biologischer Bürgerkrieg“, S. 307.

${ }^{36}$ Kluge, „Die Lücke, die der Teufel läßt“, Die Lücke, S. 259-260. Die folgenden Zitate entstammen, soweit nicht anders bezeichnet, dieser Erzählung.

37 Joseph Vogl, „Kommentar zu ,Die Lücke, die der Teufel läßt‘“, in Thomas Combrink (Gastredaktion), Text \& Kritik. Alexander Kluge, 85/86, 2011, S. 120-124.

38 Ibid.
} 
Aufhebung der aristotelischen Trennung von poetischer Dichtung und historischer Erzählung) frei von Herrschaftsanspruch und Hierarchie ist, und der Erzählung schließlich einen utopischen Aspekt verleiht.

Der literarischen und auch komischen Qualität der Erzählung, die bei der Lektüre dieses theoretisch dichten Kommentars hinter der Interpretation zurückbleibt, sei nun ein Nachsatz gewidmet. Auf die Immaterialität des menschlichen Knies „als bewegtes Dazwischen des Verhältnisses“, wie es im schwebenden Echo der Paronomasie „da ich verbinde und nicht bin“ ${ }^{39}$ anklingt, hat $u$. a. Herbert Holl in „Die Gewalt des Zusammenhangs. Kortex und Oberschenkelhalsknochen " ${ }^{40}$ hingewiesen: „das Knie, das man nur in der Lücke des unbeschreibbaren Zusammenhanges von Ober- und Unterschenkel sieht". Zu diesem eigensinnigen, entmaterialisierten aber unablässig redenden Knie, dem Kluge in Die Patriotin ${ }^{41}$ ein filmisches Denkmal gesetzt hat, gesellt sich in der Erzählung „Die Lücke, die der Teufel läßt“ ein zweiter Charakterdarsteller aus Christian Morgensterns Galgenliedern, und zwar gleichfalls als anwesend-abwesend: das Mondschaf. Genannt wird es nicht, doch der Titel des Kapitels „Die Mondkräfte und der Endsieg“ enthält bereits einen Teil des phantastischen Tiers. Der erste Satz: „Dr. Ebner (Planities) stand dem Ingolstädter Kollegen Eckholt feindlich gegenüber" ${ }^{42}$ greift Morgensterns in planitie auf, die Ebene, in der auf weiter Flur bei Morgenstern das Mondschaf steht - und wirft damit ein besonderes Licht auf die Ingolstädter Gerichtsszene: „Lunovis in planitie stat/ cultrumque magn' expectitat./ Lunovis“" 43 .

Während Dr. Ebner also den Ehrennamen ,weite Flur“ trägt, steht, gewissermaßen darin, auch wenn es vermag, sich einen Halm herauszurupfen, bleich das Mondschaf der modernen Lyrik. Bei Morgenstern hält es sich für das Universum und liegt doch in der vierten Strophe tot darnieder. Bei Kluge handelt es sich um zwei Widersacher mit Alleingeltungsanspruch menschlicher Art, Dr. Eckholt und Dr. Ebner, doch wem kann eine „weite Flur“, eine Ebene, „feindlich“" gegenüberstehen?

Übrigens erscheint Ebner, der sich um die Freilassung der Angeklagten bemüht, zunächst als die verständnisvollere Arztfigur. Vor dem Inquisitionsgericht beschränkt sich seine Argumentation jedoch auf Beschreibung und Verallgemeinerung der beobachteten Phänomene (,ein Wundschock“ trete „,immer spontan“ ein). Seine naturwissenschaftliche Herangehensweise sieht in der angeklagten Frau nur ein Exemplar der Gattung und ihr Weinen wird als Phänomen ohne „Ausdrucksqualität, gesehen vom Bewußtsein“ beurteilt. Dieses, da es „keinen verbalen, schriftlich bezeichenbaren Inhalt" hat, sei kreatürlichen Ursprungs, und insofern für das Urteil bedeutungslos. Es kündigt sich in dieser Sichtweise, hier noch bezeichnet

39 Kluge, Die Patriotin, Frankfurt a. M., Zweitausendeins, 1979, S. 248.

${ }^{40}$ Herbert Holl, „Die Gewalt des Zusammenhangs. Kortex und Oberschenkelhalsknochen“, in Christian Schulte/Rainer Stollmann (Hrsg.), Der Maulwurf kennt kein System. Beiträge zur gemeinsamen Philosophie von Oskar Negt und Alexander Kluge, Bielefeld, Transkript, 2005, S. 131-152, v.a. S. 147.

${ }^{41}$ Die Patriotin (1979), in Alexander Kluge, Sämtliche Kinofilme, DVD 10, Zweitausendeins, 2007.

42 Kluge, Die Lücke, S. 295.

43 „Das Mondschaf steht auf weiter Flur/ Es harrt und harrt der großen Schur./ Das Mondschaf.“ Christian Morgenstern, Werke und Briefe, Stuttgarter Ausgabe Bd. 3: Humoristische Lyrik, hrsg. von Reinhardt Habel et al., Stuttgart, Urachhaus, 1990, S. 63. 
als die eines „entfernte[n] Schüler[s] des Paracelsus“, bereits das cartesianische Menschenbild an. Der kritische Eckholt hingegen, unsympathisch und von seiner Anklage besessen, inquisitorisch und darüber hinaus leicht zu kränken, stellt Fragen, die durchaus an spätere psychoanalytische Herangehensweisen erinnern und über den naturwissenschaftlichen Determinismus hinausweisen. Zwei solchermaßen opponierende literarische Arztgestalten, nämlich Hofrat Prof. Behrens und Dr. Krokowski, finden sich auch in Thomas Manns Der Zauberberg. Gegenüber dem Klinikchef verficht der psychoanalytisch denkende Kollege die entscheidende Rolle des Unbewussten. Vierhundert Jahre zuvor könnte sich das in der Tat so angehört haben, wie es Kluge Eckholt in den Mund legt: „, Geschrei mit Tränenfluß ${ }^{6}[\ldots]$ sei irgendwie auszudeuten entweder als Geständnis oder als Leugnung“"

Ebner ließe sich in der Figurenkonstellation jedoch auch dadurch charakterisieren, dass er, indem er eine Horizonterweiterung auf das nicht eingegrenzte, offene und riskante Gebiet der Natur wagt, quasi als nüchtern-pragmatisches Fundament zur Verfügung steht. Seinen Thesen schließt sich der Richter, ,privat ein Liebhaber der Metamorphosen des Ovid“, zwar nicht an, sie eröffnen ihm jedoch die Möglichkeit, in einem ausgeklügelten Gedankenspiel eine „Lücke“ in der festgefügten Anklagekonstruktion zu finden. Letztlich bleibt unausgesprochen, welches Bild von Verfolgung dem Richter vor Augen gestanden haben mag, denkbar wäre Daphnes grausame und schutzbietende Verwandlung in einen Lorbeerbaum. Da in der Disputation weder die Position des fanatischen Eckholt noch die rationale Erklärung Ebners zu einer Entscheidung führen, gelingt es dem Richter über einen literarischen Umweg aus dem (Be)deutungszwang herauszufinden. Paradox mag scheinen, dass ausgerechnet sein reduzierter Anspruch an die Wahrheitsfindung, der sich lediglich auf die negative Vorannahme, es gebe kein Hindernis für einen Freispruch, stützt, die größte Reichweite hat, nämlich so, dass er die von Anfang an als „Halbhexe“ titulierte, vorverurteilte „Delinquentin“, „Novizin des Teufels“ freisprechen kann. Weil deren Weinen paradoxerweise als „unentscheidbares Zeichen“, das man „so oder so deuten“ kann, bezeichnet wird, liefert es den Schlüssel für eine von Kluge geprägte Wendung von der „Befreiung des Ausdrucks vom Zwang des Sinns“44. Die Möglichkeit, dass Literatur auch mit der sinnfernen Dimension der Sprache spielt, ist übrigens schon im ersten Satz enthalten, denn dass eine planities, eine plane Fläche, überhaupt „stehen“ soll, führte zu einer so sinnlosen Metapher, wie die schräge Vorstellung, dass die norddeutsche Tiefebene den Alpen feindlich gegenüber ,stünde““.

\section{Schlussfolgerung}

Letztlich interessiert sich Alexander Kluge nicht so sehr dafür, historische oder literarische Erscheinungsformen des Guten oder des Bösen, bzw. des dem Bösen sich Widersetzenden darzustellen. Wesentlich ist seinen Texten vielmehr, dass mit Hilfe der Erzählungen Gut und Böse, Reinheit und Teufelsbesessenheit, Transgression

${ }^{44}$ Kluge, Primetime (13.6.1999), zit. nach Christian Schulte, „Cross-Mapping. Aspekte des Komischen“, in Schulte/Stollmann (Hrsg.), Der Maulwurf, S. 224. 
und Rettung ${ }^{45}$ als potentiell ununterscheidbar erkannt werden können. Im vierten Kapitel wird, nach den historisch-theologischen Geschichten, die Frage nach dem Bösen und der Bösartigkeit bis hin zu Fragen der Forschung in NS-Laboren, der „fundamentalistischen Weltbewegung“ 46 vorangetrieben. Dazu gehört die Beobachtung des Phänomens, dass die Suche nach dem Bösen aus den suchenden Agenten ihres Ziels macht. Obwohl in einem Fall, bei der kriminalistischen Untersuchung eines Serienmörders ${ }^{47}$, diese Forschung ursprünglich vom Wunsch nach Erkenntnis geleitet zu sein scheint, schlägt sie rasch um und endet mit der Ermordung des Mörders im Labor. Die Frage bleibt offen, ob sich jenseits von Gedankengebäuden, seien sie „Wissenschaft als Glaubenskunst“"48, Aberglauben oder Logik, brauchbare Maximen zum Handeln finden lassen. Mag auch der Widerspruch benannt werden, z. B. „logisch war es nicht“"49, das Handeln richtet sich im Einzelfall nicht danach. Verfolgt man jedoch beispielhaft nur diesen einen thematischen Schwerpunkt, lässt sich zeigen, dass Kluges Vorschläge zur navigierenden Lektüre auch noch eine dritte, Karten und Routen transzendierende Dimension enthalten. Nicht ohne Metaphysik, noch ohne Logik, und darüber hinaus mit einer „historischen wie politischen Topografie“ ${ }^{\text {50 }}$ ausgestattet, das könnte als Motto für Kluges Die Lücke, die der Teufel läßt gelten. Im Unterschied zum Philosophen Adorno versucht sich Kluge jedoch nicht an Darstellung und Kritik des „Verblendungszusammenhangs“, entwirft keine Negative Dialektik ${ }^{51}$, sondern baut, unter Ausgestaltung des schwachen Arguments, auf singuläre Ereignisse, Zweifel, Brüche, kluge Ironie und Dada. Diese, nicht die Kraft des Arguments, Glauben bzw. Sicherheit, oder Machtverhältnisse, sind die Momente, an denen die objektive Macht der Zeichen endet. Alexander Kluges Satz von 1966 „Wahrheit, wenn sie zu ernst auftritt, wird totgeschlagen" wirft einen Abschiedsblick auf gestern ${ }^{52}$. In Die Lücke, die der Teufel läßt werden mit dem Satz „Wenn doch die Wahrheit zerstreut sein kann in sämtlichen Texten!“ ${ }^{53}$ diese für die Zukunft gerettet. Sie auf weiter Flur aufzulesen, steht allen frei.

${ }^{45}$ Kluge, „Es ist gleich, unter welcher Oberfläche sich eine tugendhafte Tat verbirgt“, in Die Lücke, S. 694.

46 Ibid., S. 298.

${ }^{47}$ Kluge, „Fund eines Wildtyps des ,verbrecherischen Menschen““, in Die Lücke, S. 311.

48 Kluge, Die Lücke, S. 298.

49 Kluge, „Fund eines Wildtyps“, in Die Lücke, S. 313.

${ }^{50}$ Vogl, „Kommentar“, S. 120.

51 Theodor W. Adorno, Negative Dialektik, Frankfurt am Main, Suhrkamp, 1966. Siehe darin u.a. S. 397.

${ }^{52}$ Abschied von gestern (1966), in Alexander Kluge, Sämtliche Kinofilme, DVD 1, Zweitausendeins, 2007.

${ }^{53}$ Kluge, „Dem Himmel bin ich auserkoren“, in Die Lücke, S. 270. 